\title{
Collagen degradation
}

\author{
DAVID J. ETHERINGTON \\ From the ARC Meat Research Institute, Langford, Bristol
}

The native collagen fibre is extremely resistant to proteolytic degradation at physiological pH. Resynthesis of collagenous structures in the adult animal proceeds at a very low rate and attempts to determine the half-life values have shown that in some rat tissues these extend beyond one year (Pierce et al., 1964). Under certain physiological conditions previously stable collagen fibres are rapidly removed by specific enzymes which appear to operate in a selective and conservative manner.

The different physiological events in which collagenolysis occurs have been grouped by Woessner (1968) into three classes-(1) normal, (2) pathological, or (3) induced. Normal resorption of collagen occurs, for example, during tissue remodelling with growth, especially embryogenesis, when there is an overall net synthesis of new fibrous tissue. A net loss of collagen occurs specifically with involutionary processes, and the uterus after parturition provides a most extreme model for studying collagen resorption. In rats half the uterine collagen is lost in about 24 hours post partum and the process is completed in 4-6 days. Abnormal collagen resorption occurs in various pathological disorders such as Paget's disease, rheumatoid arthritis, and scurvy. The degradation of collagen is induced in response to trauma such as skin wounds, burns, or bone fractures and forms an essential part of the healing process.

\section{Collagenases}

All initial attempts to identify an enzyme in tissue extracts that might resemble the bacterial collagenase were unsuccessful, and it was only when tissue explants were grown in culture that the existence of an animal collagenase was first established. This enzyme, which was detected in cultures of the tadpole tail at metanorphosis (Gross and Lapiere, 1962), was subsequently purified and studied in detail (Nagai et al., 1966). The tadpole enzyme, unlike bacterial collagenase, was shown to attack the triple-helix at a single locus which was $25 \%$ of the length from the C-terminal end of the molecule. Further peptide cleavage occurred only when these two less stable portions of the collagen molecule were allowed to denature thermally at $37^{\circ} \mathrm{C}$. In other properties the two enzymes were similar- calcium was required as an activator and the optimal $\mathrm{pH}$ range for lysis was 7-8.

The use of culture techniques was extended to the investigation of other tissues. The existence of a neutral collagenase has now been established in virtually every tissue in which there is active collagenolysis. An extensive literature now exists, particularly on the mammalian collagenases, and a number of excellent reviews have appeared (Eisen et al., 1970; Nordwig, 1971; Woessner, 1973; Lazarus, 1973; Harris and Krane, 1974). Every enzyme examined so far is activated by calcium, and at temperatures below $30^{\circ} \mathrm{C}$ these collagenases cleave the same site in the collagen molecule as the tadpole enzyme to yield $\frac{1}{4}$ and $\frac{3}{4}$ fragments from reconstituted fibrils. At $37^{\circ} \mathrm{C}$ smaller peptides are released as these fragments spontaneously denature (Gross, 1970). The ability to cleave all three chains of the triple helix at a single locus is a uniques property of the animal collagenases. In type $\mathrm{I}$ collagen the susceptible peptide bond in the $\alpha 1$ chain is located at $\mathrm{Gly}_{722}-\mathrm{Ile}_{\mathbf{7 2 3}}$ and at the homologous Gly-Leu bond in the $\alpha 2$ chain (Gross et al., 1974). The three identical $\alpha 1$ chains in type II collagen are cleaved at the same homologous Gly-Ile bond, and the three type III $\alpha 1$ chains at Gly-Leu (Miller et al., 1976). Highly purified collagenase exhibits no detectable proteolytic activity against any other potential substrate examined so far (Woolley et al., 1975a).

A wide variation persists in the molecular weight values for the different collagenases. This was originally believed to arise from the formation of oligomers (Harris et al., 1969) but it now seems certain from studies of the human enzymes that several different molecular species are produced (Woolley et al., 1976).

Collagenase is readily complexed by serum proteinase inhibitors, in particular $\propto 2$ macroglobulin and $\alpha 1$ antitrypsin. However, recently a slightly smaller serum protein than $\alpha 1$ antitrypsin has been shown to be the potent inhibitor of the human collagenases rather than $\alpha 1$ antitrypsin itself (Woolley et al., 1975c, 1976). These proteins are effective in removing active enzyme from solution during tissue extraction and this has always precluded a direct assay for the enzyme. Radioimmunoassay of collagenase has been attempted 
(Bauer et al., 1972) but has not been widely adopted. Secreted collagenase binds tightly to the endogenous collagen fibres under physiological conditions and an attempt has been made to quantify the enzyme in the collagenous debris by autolysis after extraction of soluble proteins (Ryan and Woessner, 1971). Alternatively, the enzyme can be dissociated and recovered from its natural substrate by the use of strong salt solutions (Wirl, 1975). The standard assay for collagenase in culture medium has been to measure the rate of lysis of ${ }^{14} \mathrm{C}$-labelled reconstituted fibrils of soluble rat or guinea-pig skin collagen. The method is sensitive and reproducible, but this form of the substrate does not normally exist in vivo.

Initial attempts to demonstrate degradation of the cross-linked native fibres were unsuccessful (Lieberwich and Weiss, 1971; Harris and Farrell, 1972), and this was owing to the use of insufficient enzyme in the assays. When rabbit fibroblast collagenase (Werb and Burleigh, 1974) and rheumatoid synovial collagenase (Woolley et al., 1975a) were present in adequate concentration a slow but measurable breakdown of insoluble type I collagen was demonstrated. The different genetic forms of collagen do not exhibit equivalent susceptibility to collagenase. Soluble type $\Pi$ collagen from lathyritic cartilage proved to be inferior to soluble type I collagen as a substrate for the rheumatoid synovial collagenase by a factor of six (Woolley et al., 1975b) and soluble type III was similarly more susceptible than type $\Pi$ collagen to rabbit tumour collagenase (Miller et al., 1976).

\section{Collagenolytic activity of other proteinases}

The granulocyte neutral proteinases have been studied in detail in recent years and an elastase (Feinstein and Janoff, 1975a; Starkey and Barrett, 1976a, b) and chymotrypsin-like enzyme (Feinstein and Janoff, 1975b; Starkey and Barrett, 1976a, c) have been purified and characterized. Crude extracts of these enzymes can degrade insoluble collagen and may have an important synergistic action with the granulocyte collagenase (Stevens et al., 1975). On testing the purified enzymes Barrett (personal communication) has found that the elastase efficiently degrades monomeric collagen in the telopeptide region, which carries the intramolecular cross-links.

Whereas neutral collagenase is not immediately identifiable in tissue extracts, the endogenous lysosomal cathepsins can be readily shown to degrade either soluble or insoluble collagen in an acidic medium (Frankland and Wynn, 1962; Anderson, 1969). Maximal solubilization of fibrous collagen occurs at $\mathrm{pH} 3.5$ in a sharp peak of activity. Below $\mathrm{pH} 2.5$ and above $\mathrm{pH} 4.5$ the substrate remained intact. This striking response to pH can be interpreted from the swelling effect of acid on the collagen fibril, which allows the enzymes to gain access to the susceptible peptide bonds only at the lower end of the normal range for proteolysis by these lysosomal enzymes (Etherington, 1974). This observation was confirmed from studies on soluble collagen when an enhanced rate of degradation was observed at pH 4-5. Cathepsin B1 was purified from spleen (Etherington, 1974) and liver (Burleigh et al., 1974) and shown to have collagenolytic activity. The principal site of cleavage in native collagen was in the cross-linking nonhelical telopeptide region. Denatured collagen is further degraded to low molecular weight peptides (Burleigh et al., 1974).

Another lysosomal enzyme, tentatively known as collagenolytic cathepsin (Bazin and Delaunay, 1970; Etherington, 1974) has been partially purified and characterized. It requires the same thiol activators as cathepsin B1 and degrades collagen in a similar manner, but mixtures of the two enzymes exhibit a synergistic effect with insoluble collagen as the substrate (Etherington, 1976).

\section{Mechanisms of collagenolysis}

Histological studies of tissues where there is active resorption of collagen have in certain instances
clearly revealed the existence of ultrastructurally intact fragments of collagen in the endocytic vacuoles of macrophages (Brands and Anton, 1969; Parakkal, 1969, 1972; Sambe et al., 1974). In the involuting uterus these macrophage vacuoles are abundant and appear within two hours after parturition. Fusion then occurs with acid phosphatasecontaining primary lysosomes to produce the digestive vacuole, or phagolysosome, and the sequestered collagen fragments are then seen to disintegrate.

Current ideas on the mechanism for collagen resorption now support the hypothesis of a multistep process in which the two groups of enzymes can digest the collagen in a separate but sequential manner. A two-stage process was first proposed by Fell (1969) for the degradation of cartilage matrix and this concept has been developed by others to describe specifically in-vivo collagenolysis (Harris and Krane, 1974). Initially the collagen fibre, which is many times the length of a single cell, is split and fragmented in the extracellular compartment by collagenase and possibly also by the tissue elastase which are secreted directly onto the fibre by a closely apposed cell. The fragmentation of the fibre may follow through regions where the cross-links are fewer in density, and hence present lines of weakness, or, conversely, the rapid inactivation of collagenase by serum inhibitors may make this 
extracellular step a random process. The ultimate effect is to reduce the fibre to fragments which are of a manageable size for the tissue macrophages. The macrophages then endocytose the collagen fragments in the next step and digestive vacuoles are formed in the cytoplasm by fusion with the primary lysosomes. Endocytosed material has been observed with certainty in only a few tissues. This possibly reflects efficient intracellular digestion with rapid dissolution of these collagen fragments rather than a lack of phagocytic activity on the part of the macrophage.

In recent work on the periodontal ligament fibroblasts have been observed to engulf collagen fibrils in a novel manner (Ten Cate and Deporter, 1975). The cell internalizes the end of an intact fibril which it then appears to sever by producing a local secretion of collagenase on to the fibril at the point where it enters the cell. When the enzyme has digested its way through the fibril the neck of the invagination in the cell membrane then closes and the vacuole so formed, together with the sequestered collagen, then passes into the interior of the cell where it fuses with the primary lysosomes.

The second, intracellular, stage of collagen digestion which occurs in the phagolysosomes of the tissue macrophages (Brands and Anton, 1969; Parakkal, 1972) or fibroblasts (Ten Cate and Deporter, 1975) is accomplished through the action of the lysosomal cathepsins. The principal enzymes to solubilize the residual fibrous collagen are cathepsin B1 and collagenolytic cathepsin, and in this acidic environment at $37^{\circ} \mathrm{C}$ the triple helix denatures as the fibre is progressively depolymerized. The full complement of lysosomal protease then become effective to complete the breakdown of the collagen chains to amino-acids or small peptides.

Except for the fibroblasts of the periodontal ligament it has not been observed whether the two digestive stages, extracellular and intracellular, are directed by the same population of cells. The fibroblasts of the periodontal ligament appear so far to have a unique double function in that they not only degrade the collagen at this site but also undertake its resynthesis (Ten Cate and Deporter, 1975).

Cell culture studies have been initiated to identify which cell lines can synthesize collagenase. Fibroblasts from various rabbit tissues readily secreted collagenase which appeared to be identical to the enzyme released into the medium by whole tissue explants (Werb and Burleigh, 1974; Werb and Reynolds, 1975). These cells can also secrete a neutral proteinase which may act synergistically with the collagenase (Werb and Reynolds, 1974). However, attempts to produce collagenase from cultured human or mouse fibroblasts have so far been unsuccessful.

PMN leucocytes, which exist in many inflammatory conditions, have been isolated and a collagenase readily located in their granule fraction, which has prompted detailed studies of this enzyme (Lazarus et al., 1968; Lazarus, 1973). Furthermore, these cells contain neutral proteinases, including the collagen-degrading elastase (Feinstein and Janoff, 1975a), which together with collagenase appear to be associated with a non-lysosomal secretory granule. On being secreted from the cell these enzymes can degrade the extracellular supporting tissues. The same cells are then believed to endocytose the partially-degraded material and to complete the digestion intracellularly (Robertson et al., 1972). However, the data from these in-vitro investigations of PMN leucocytes cannot be assumed to provide an adequate picture of events in vivo. In particular, the leucocyte collagenase is noticeably different from the collagenase produced in culture by whole tissue explants in that whereas these enzymes are inhibited by serum the leucocyte enzyme is not inhibited. Furthermore, during purification the leucocyte enzyme loses its ability to degrade the fibrillar form of collagen and can cleave only soluble monomers (Lazarus, 1973), which implies that this collagenase by itself has little value in vivo unless the neutral proteinase is also present to cleave the cross-linking regions in collagen.

Macrophages have been identified as functionally important cells in the resorption of collagen in vivo,

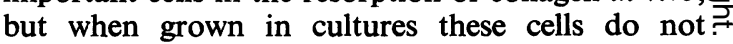
normally secrete a collagenase unless stimulated to become phagocytic. However, recent studies have revealed most convincingly that if these cells are thus stimulated they synthesize and secrete into the medium large quantities of collagenase (Wahl et al., 1974). This collagenase, unlike the PMN leucocyte enzyme, seems to be identical to the collagenase secreted by tissue explants (Werb and Gordon, 1975).

The extent and effectiveness of the extracellular, first-stage digestion of collagen at resorption is difficult to assess. As yet no suitable biological model exists which adequately allows the separate investigation of the extracellular and intracellular events in collagenolysis and which might permit the unequivocal assignment, or exclusion, of a functional role for the secreted elastase and other neutral proteinases.

Collagenase is believed to be synthesized and secreted in minimal quantities only at the site where it is required. An excess of enzyme could be particularly damaging if it diffused out and caused indiscriminate attacks on adjacent tissues (Gross, 1970). The enzyme is synthesized as an inactive zymogen, procollagenase, and is activated extracellularly in a complex, multistep manner (Vaes, 1972). Recent studies of cultured tissue explants have now shown that only part of the enzyme is 
normally in the activated form, and a short exposure to trypsin is required to activate the remaining proenzyme (Birkendal-Hansen et al., 1975). Inactivated procollagenase has also been identified in vivo in some arthritic conditions (Nagai et al., 1975). The activation mechanism for procollagenase is mediated by tissue proteinases of which lysosomal cathepsin B1 has been identified as a potential activator below pH 7. The extracellular enzymes plasmin and kallikrein can also activate procollagenase and at physiological pH (Vaes and Eckhout, 1975).

Once secreted from the cell the active enzyme binds tightly to its substrate under the normal physiological conditions of $\mathrm{pH}$ and ionic strength and therefore must act locally. Any free collagenase is rapidly and irreversibly removed by the serum proteinase inhibitors. In-vivo studies in the dog have confirmed that the clearance of the PMN leucocyte collagenase and elastase proceeds at a rapid rate (Ohlsson and Delshammar, 1975).

The physiological resorption of collagen which occurs in normal or induced situations is an integrated process but the overall mechanism of activation, direction, and control of collagenolysis is so far poorly understood. Recent work on the postpartum involution of the uterus has shown that there is a strong hormonal dependency which can be readily blocked (Ryan and Woessner, 1974; Jeffrey et al., 1975). Other situations which involve developmental changes are probably also subject to the overriding action of humoral agents. During wound healing and in other locations where the resorption is of an induced nature, the stimulus for collagenolysis occurs locally within the traumatized area. Initially, at least, this stimulus causes macrophage activation (Wahl et al., 1974; Werb and Gordon, 1975) and is closely related to the development of the inflammatory reaction. The pathways for activation of newly secreted procollagenase also interconnect with the pathways for the formation and action of kinins in the inflamed area (Vaes and Eckhout, 1975). After wound healing the scar tissues occasionally become hypertrophic or there is keloid formation. In these circumstances there is abundant and continuous synthesis of new collagen which does not form mature and stable fibres but is subsequently degraded (Bailey et al., 1975a). A similar situation has been observed in the implanted sponge granuloma (Bailey et al., 1975b) and the factors which promote the continued turnover of collagen have not been elucidated.

The lysosomal enzymes can be secreted from the cell to act in the extracellular space, and these events have been well documented for the lysosomal degradation of the proteoglycan moiety of cartilage matrix (Barrett, 1975; Poole, 1975). However, it has not been established whether extracellular collagen can be similarly degraded by the direct action of secreted cathepsins, although there has been much speculation in the past for such a mechanism (Woessner, 1968). The major difficulty arises from the need to achieve an acidic environment for these enzymes in the extracellular space, since they are active only at low $\mathrm{pH}$. During intracellular digestion the macrophage generates and maintains acidic conditions within the phagolysosome near $\mathrm{pH} 5$ through an energy-linked process (Mego, 1973). The erosion of bone matrix by the osteoclast is a process which occurs extracellularly, and Vaes (1969) has provided a detailed review on this topic. This cell forms a fluid pocket between itself and the bone surface into which it secretes lysosomal enzymes. This micro-environment is apparently controlled by the cell itself and a good correlation was observed between the extent of bone resorption and the extracellular levels of specific lysosomal enzymes. Clearly an acidic medium is unfavourable to the efficient action of the bone collagenase although it may be expected to be readily formed from activation of procollagenase by extracellular cathepsin B1.

The circumstances pertaining at the surface of bone where the osteoclast has a unique control overt. this extracellular region pose several questions in relation to the resorption of the collagen moiaty from bone. Collagenase and the neutral proteinases are inactive below about $\mathrm{pH} 5.5$ and in an acidic environment these enzymes would be ineffective. Either the $\mathrm{pH}$ is varied to permit the sequential action of the neutral and acidic groups of enzymes or they must all act together in what would appear to be compromise conditions.

A similar situation seems to exist between the growing pannus and the surface of articular cartilage during the development of rheumatoid arthritis. The microenvironment which exists here beneath the pannus is again controlled by the cells of this tissue. Serum proteins are also excluded from this site and the erosion of cartilage matrix can therefore proceed unimpeded, owing to the body's inability to provide a blockade from its own circulating inhibitors. Studies on the collagenolytic system in rheumatoid arthritis have shown a strong link between the production of a neutral collagenase and the loss of collagenous structure (Harris et al., 1975a; Lane and Weiss, 1975), but within this narrow region the extracellular events must undoubtedly cause the different collagenolytic enzymes to interact in a manner which is more complex than previously envisaged. 